\title{
Memory Impairment in AB (25-35) and D-Galactose Induced Alzheimer's Disease Model Rat
}

\author{
Feijuan Huang ${ }^{1,2,3}$, Fang Bai', 3, Mengzhou Xie ${ }^{4}$, Xianxiang $\mathrm{Tian}^{3}$, Weiping $\mathrm{Li}^{2}$,

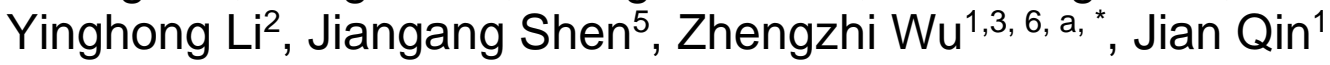 \\ ${ }^{1}$ Sun Yat-sen University First Affiliated Hospital, Guangzhou, China. \\ ${ }^{2}$ The Second People's Hospital of Shenzhen, Shenzhen University First Affiliated Hospital, \\ Sungang West Rd. 3002, Futian District, Shenzhen, China.
}

${ }^{3}$ Shenzhen Institute of Gerontology, No.1017 Dongmen North Road, Luohu District, Shenzhen China.

${ }^{4} 2011$ Collaborative Innovation Center of Digital Chinese medicine of colleges and Universities in Hunan Province, No. 300, Xueshi Road, Yuelu District, Changsha, China.

${ }^{5}$ School of Chinese Medicine, University of Hong Kong, 10 Sassoon Road, Hong Kong, Hong Kong SAR, China.

${ }^{6}$ The Futian Affiliated Hospital of Guangdong Medical Institute, Shenzhen, China.

a himyhox@yeah.net

Keywords: Alzheimer disease; Beta amyloid; neuronal cells damage in the brain.

\begin{abstract}
Beta amyloid (A $\beta)$ is a key pathological hallmark of Alzheimer disease (AD) and plays key roles the progressive process of this disease. However the involved biological processes or pathways that triggered by $\mathrm{A} \beta$ in the progress of $\mathrm{AD}$ are still unclear. To answer this question, $\mathrm{A} \beta_{25-35}$ was bilaterally injected into the rats' bilateral hippocampus and to accelerate the brain aging, d-gal was subcutaneously administrated, so as to set up the combined AD model rats and further explore the gene expression profile of the rats. The results demonstrated that the d-gal and $A \beta$ treatment led to learning and memory impairment in behavioral test and neuronal cells damage in the brain, which validated the establishment of $\mathrm{AD}$ model rats. These verified genes may provide a new insight for elucidating $\mathrm{A} \beta$ cascade hypothesis in $\mathrm{AD}$ and its progression.
\end{abstract}

\section{Introduction}

Alzheimer's disease (AD), characterized by cognitive decline and memory deficits, represents the most popular age-associated progressive neurodegenerative disease [1], accounting for about 2/3 cases of all the elderly patients with progressive cognitive impairment [2]. Although AD results in devastating effects on patient behavior and eventually poses great threat to their lives, the mild AD cases are rarely diagnosed; thus, early interventions are not practically applicable. Clinically pathological diagnosis is only available for the moderate and final stage $\mathrm{AD}$, based on the presences of extracellular amyloid plaques (APs) and intracellular neurofibrillary tangles (NFTs) in the cerebral cortical and hippocampal regions of the patients [3]. Exploration and development of useful biomarker for the early diagnosis of $\mathrm{AD}$ has been the targets for the investigators over the world for many years.

The amyloid plaques (APs) are comprise of amyloid $\beta$ peptides, short amino acids fragments ( 40-43 amino acids), produced by sequential cleavage of amyloid precursor protein (APP) by $\beta$ - and $\gamma$-secretase $[4,5]$. Many previous studies demonstrate that the amyloid $\beta$ peptides play curial roles in the gradual progressive process of AD since the accumulation and aggregation of amyloid $\beta$ peptide result in oxidative damage as well as the further neuronal apoptosis/death [6]. Many previously identified AD cases were shown an increased deposition of $\beta$-amyloid in brain $[7,8]$. A $\beta$ preserve impaired cognition in the patients with $\mathrm{AD}$, however, a cascade of molecular events and biological pathways triggered by $A \beta$ in this neurodegenerative process remain obscure. 
At present, the application of animal model to investigate involved biological pathways of $A \beta$ is increasing and many previous studies have successfully set up $A \beta$ related or $A \beta$ induced-AD models to study the fundamental mechanisms of AD. The senescence-accelerated mouse prone 8 (SAMP8), a substrain of the senescence-accelerated mouse through phenotypic selection, is an A $\beta$ related-AD models, showing age-dependent learning and memory deficits, and $A \beta$ accumulation [9]. The human double mutant APP/PS-1 knock-in mice, which harbor a PS-1 mutation, found in familial AD and a mutation in the APP gene, is an A $\beta$-induced transgenic AD model with the increasing oxidative stress and $A \beta$ expression in the brain $[10,11]$. In current study, to investigate the role for $A \beta$ in the pathogenesis of AD and to avoid other altered transcriptional level induced by exogenous genes in the transgenic $\mathrm{AD}$ rats, the $\mathrm{A} 325-35$ was directly injected into the rats' bilateral hippocampus. Additionally, it has been reported that d-galactose induced behavioral and neurochemical changes that could mimic many characteristics of the natural process of brain aging [12]. Thus, combined with Aß25-35 injection, d-gal was subcutaneously injected to set up the AD rat model, which may provide hints for elucidating $A \beta$ and d-gal induced $A D$ model rats and shed light on the discovery of the novel biomarkers for Alzheimer's disease.

\section{Materials and Methods}

\subsection{Experiment Design}

A controlled parallel experiment was performed so as to compare the gene expression profiles between the AD model group and the controlled sham-operation group.

\subsection{Methods}

\subsubsection{Experiment Design and Establishment}

A total of 24 male Sprague Dawley rats, age of 12-14 weeks, weighing (255 \pm 15$)$ g, purchased from the Laboratory Animal Center of Guangdong Province (Certification No. SCXK (Yue) 2008A020), were assigned to AD model group, sham-operation group and normal group (8 rats per group). After 2 week's adaptive feeding, according to the procedures that described previously [13], the rats were injection with pentobarbital sodium $(45 \mathrm{mg} / \mathrm{kg}$ body weight), intraperitoneally anesthetized and placed in a stereotaxic frame. The hair was shaved and the scalp was exposed. The holes with a diameter of 1-2 mm were drilled according to a mouse brain atlas (AP-3.0 mm, ML 2.0 $\mathrm{mm}$, DV2.9 mm). Then, $5 \mu \mathrm{l}(2 \mu \mathrm{g} / 5 \mu \mathrm{l})$ of the prepared aged amyloid peptides A $\beta 25-35$ (Sigma, USA) solution for one hole were injected at a slow rate $(1 \mu \mathrm{l} / \mathrm{min})$. After the surgery, each rat was fed in a separate cage with a treatment of $50,000 \mathrm{u} /$ day penicillin for 3 days to prevent infection. As a control, Sham-operation group was also performed through the injections of saline at the same volume. After 2 weeks, to generate an aging brain of rats, the d-gal was subcutaneously injected at a dose of $150 \mathrm{mg} / \mathrm{kg}$ per day for 50 days ( 7 weeks). For the sham-operation and normal group, the same dosage of saline was injected instead of d-gal.

\subsubsection{AD Model Validation}

The behavioral tests for the subjects from different groups on the 14th day post d-gal administration were assessed in a Morris water maze. The apparatus was set up as described in [14]. With the aid of the video tracking system, the escape latencies, which the rats took to escape from water to the safe platform, and the swimming distances between the start point and the end, were recorded to assess the rats' spatial recognition memory in different groups. The rats placed in the apparatus to test were according to a random order in the test.

At the end of experiments, all the rats were killed through decapitation. The left-brain was fixed with $4 \%$ paraformaldehyde and stained by cresyl violet (Nissle staining). Three slices were randomly selected from 6 slices in each group for microscopic observation (magnification of 100) and in every slice, three visual fields in the hippocampus CA2 and CA3 regions were randomly taken to calculate the average number of neurons in automatic micro-image analysis system. 


\section{Results}

\subsection{The Establishment of AD Model Rats}

Except for the subjects in AD model group, rats were gaining weight normally. The rats in the AD model group grew relatively slow (Figure 1) with normal diet and exercises. There were no obvious secretions near mouth, eyes or genital pore.

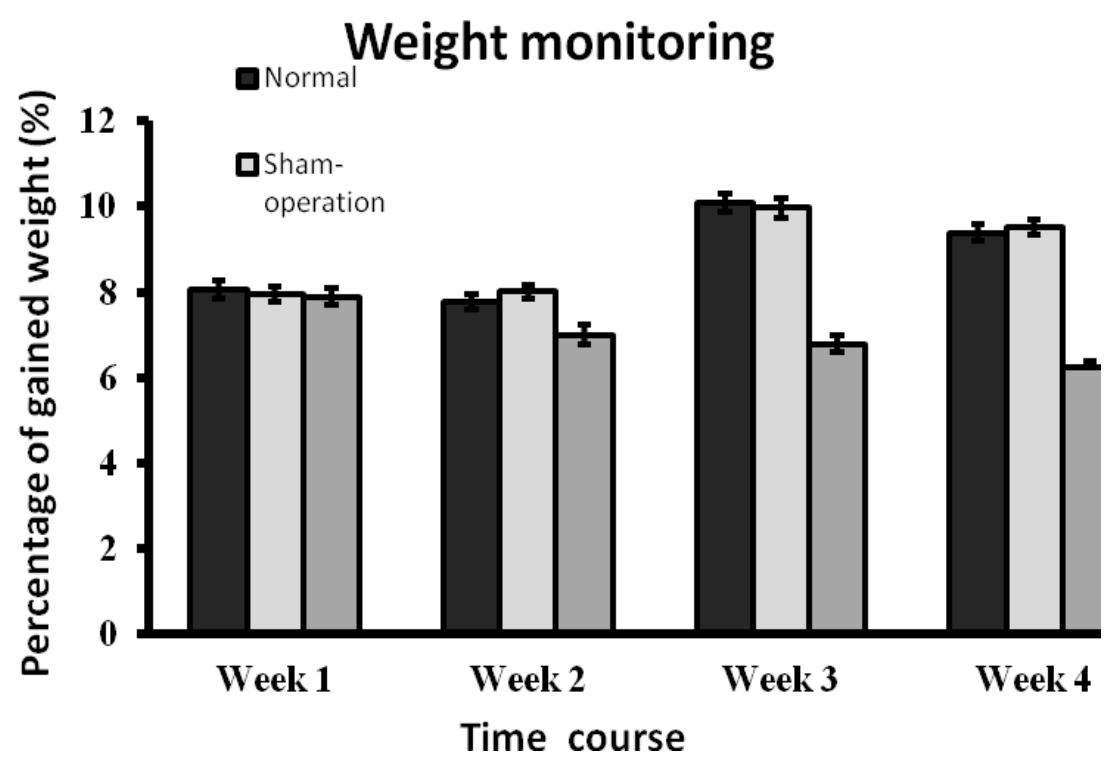

Figure 1. Body weight monitoring for mice in different groups $(n=10, *$ represents $p<0.05$, vs. sham-operation group)

Mean escape latencies and swimming distances in the single probe acquisition in Morris water maze were shown in Figure 2A ( $a$ and $b$ ) respectively. On both of escape latency and swimming distance, the differences between the control group and sham-operation group were not statistically significant, which exclude the negative effects of surgery on spatial recognition memory. The rats in AD model group presented overall longer escape latencies and distances, nearly $2 \sim 3$ fold, than those in the other two groups ( $\mathrm{P}<0.05$ in all cases), suggesting that the rats in $\mathrm{AD}$ model developed an impaired spatial memory.

As the Figure 2B demonstrated that there was no significant difference between normal and sham-operation group. There was no obviously damage in the neurons in the sham-operation group was observed, where the morphology of neurons were complete with large and clear nucleus. Neurons in the AD model group were sparse in a disordered arrangement and partially damaged, with incomplete morphology. The nucleus became small and unclear and the arrangement of neuronal fiber became disordered (Figure 2B).

The neurons in the CA2 and CA3 regions of hippocampus were counted in the 3 random visual fields in the microscope with 200 times amplification. Figure $2 \mathrm{C}$ showed the neuronal counts in each group. The number of neurons in the normal group and sham-operation were almost the equal but the relative number of neurons in $\mathrm{AD}$ group was dramatically dropped by almost $\sim 35 \%$, compared to the normal and sham-operation group. 

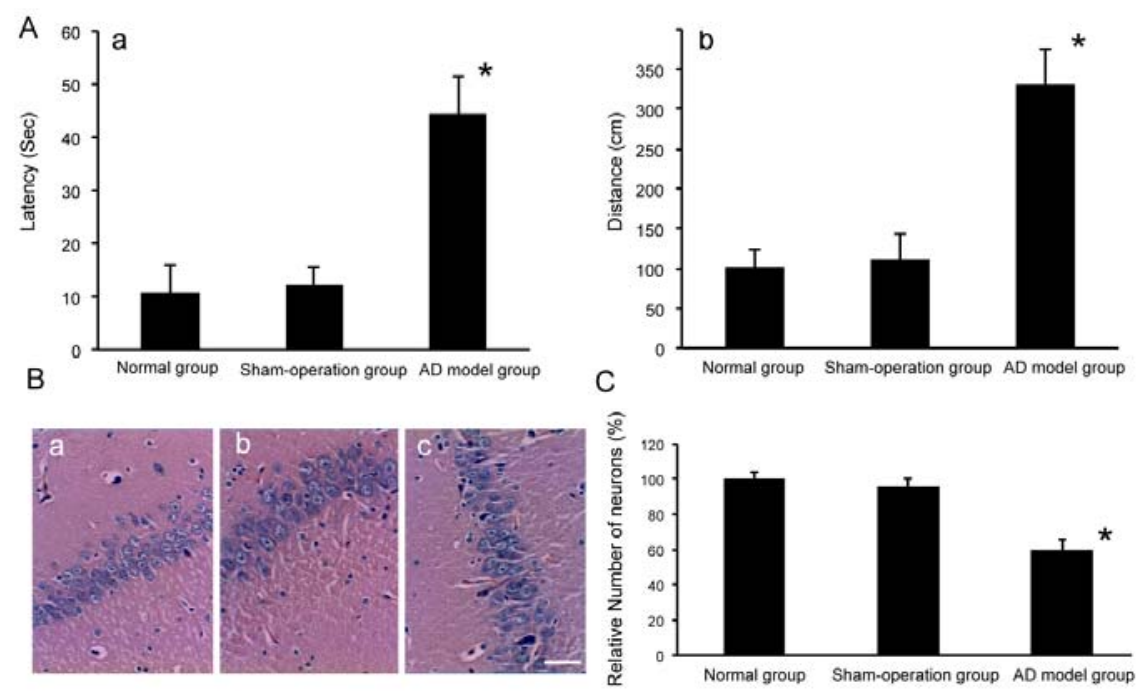

Figure 2. Differences between the AD model group, the normal and the sham-operated subjects: A Mean latencies (a) and distances (b) to reach the platform in the Morris water-maze tests. Statistical analyses, overall group effect: *significantly different from Normal and Sham-operation group, $\mathrm{P}<$ 0.05. B Nissle's staining of neurons in rats' hippocampus (a: Normal group, b: Sham-operated group, $\mathrm{C} \mathrm{A} \beta$ and d-galactose-induced $\mathrm{AD}$ mode group), scale bar: $50 \mu \mathrm{m}$. $\mathrm{C}$ Decrease of neurons density in $\mathrm{A} \beta$ group (data were obtained from six experiments counting).

Previous studies have found that $A \beta$ deposition via the expression of amyloid precursor protein gene in the transgenic models [10] or direct injection of A $\beta$ into hippocampus [15] impaired learning and memory ability, thus it is a useful method to set up the AD model. In current study, to accelerate brain aging of the $\mathrm{AD}$ rats, $\mathrm{d}$-gal was subcutaneously administered. Consistent with the results of the previous studies in $\mathrm{A} B$ injection $\mathrm{AD}$ model rats $[16,17]$ and d-gal chronic administration aging model rats [18], these AD model rats had significantly longer escape latencies and distances to reach the safe platform than those in the normal or sham-operation group, showing an impaired cognitive function. Due to the degenerative effect of $A B$ and d-gal, it has found that the density of neurons cells was decreased, with part of them showed morphological signs of cell damage in brain after the A 3 and d-gal injection, which agrees with the previous investigation [19]. Thus, the AD model rate was successful established.

Many previous studies in the $A D$ research proposed the $A B$ cascade hypothesis, in which the $A B$ triggers a cascade of molecular events leading to inflammation and oxidative stress and correspondingly resulting in neuronal dysfunction and cognitive decline [20]. However, the molecular/events involved in the $A ß$ cascade and their respective effects to the disease process are required to be identified in details. Strategies aimed to profile the transcripts expression level so as to address the corresponding biological pathways that interfered by the deposition of amyloid-beta in the brain were applied in further study.

\section{Acknowledgments}

This work was supported by Guangdong Science and Technology Foundation (2016A020215028 \& 2013B021800101) and Shenzhen Major Project of Science and Technology Planning (JCYJ20150401171519333 \& JCYJ20130401115231337).

\section{References}

[1]. Cummings, J.L. and G. Cole, Alzheimer Disease. JAMA: The Journal of the American Medical Association, 2002. 287(18): p. 2335-2338. 
[2]. Querfurth, H.W. and F.M. LaFerla, Alzheimer's disease. The New England journal of medicine, 2010. 362(4): p. 329-344.

[3]. Brayne, C., R. Mayeux, and C. Reitz, Epidemiology of Alzheimer disease. Nature Reviews Neurology, 2011. 7: p. 137+.

[4]. Lesne, S.E., et al., Brain amyloid-beta oligomers in ageing and Alzheimer's disease. Brain, 2013. 136(Pt 5): p. 1383-98.

[5]. De-Paula, V.J., et al., Alzheimer's disease. Subcell Biochem, 2012. 65: p. 329-52.

[6]. Morris, G.P., I.A. Clark, and B. Vissel, Inconsistencies and controversies surrounding the amyloid hypothesis of Alzheimer's disease. Acta Neuropathol Commun, 2014. 2(1): p. 135.

[7]. Li, Q. and T.C. Sudhof, Cleavage of amyloid-beta precursor protein and amyloid-beta precursor-like protein by BACE 1. J Biol Chem, 2004. 279(11): p. 10542-50.

[8]. Murphy, M.P. and H. LeVine Iii, Alzheimer's Disease and the Amyloid- $\beta$ Peptide. Journal of Alzheimer's Disease, 2010. 19(1): p. 311-323.

[9]. Poon, H.F., et al., Antisense directed at the A $\beta$ region of APP decreases brain oxidative markers in aged senescence accelerated mice. Brain Research, 2004. 1018(1): p. 86-96.

[10]. Webster, S.J., A.D. Bachstetter, and L.J. Van Eldik, Comprehensive behavioral characterization of an APP/PS-1 double knock-in mouse model of Alzheimer's disease. Alzheimer's Research \& Therapy, 2013. 5(3): p. 1-15.

[11]. Huang, Q., et al., Potential in vivo amelioration by N-Acetyl-L-cysteine of oxidative stress in brain in human double mutant APP/PS-1 knock-in mice: Toward therapeutic modulation of mild cognitive impairment. Journal of Neuroscience Research, 2010. 88(12): p. 2618-2629.

[12]. Woo, J.-Y., et al., Lactobacillus pentosus var. plantarum C29 ameliorates memory impairment and inflammaging in a d-galactose-induced accelerated aging mouse model. Anaerobe, 2014. 27: p. 22-26.

[13]. Chen, D.-L., et al., Protective effects of bajijiasu in a rat model of A $\beta 25-35$-induced neurotoxicity. Journal of Ethnopharmacology, 2014. 154(1): p. 206-217.

[14]. Wang, H.-M., et al., Neuroprotective effects of forsythiaside on learning and memory deficits in senescence-accelerated mouse prone (SAMP8) mice. Pharmacology Biochemistry and Behavior, 2013. 105: p. 134-141.

[15]. Li, Y., Establishment of experimental models for Alzheimer's disease research. International Journal of Neuroscience, 2013. 123(12): p. 823-831.

[16]. Fang, F. and L. Geng-tao, Protective effects of compound FLZ on $\beta$-amyloid peptide-(25-35)-induced mouse hippocampal injury and learning and memory impairment. Acta Pharmacologica Sinica, 2006. 27(6): p. 651.

[17]. Liu, R., et al., Quercetin protects against the A $\beta 25-35$-induced amnesic injury: Involvement of inactivation of RAGE-mediated pathway and conservation of the NVU. Neuropharmacology, 2013. 67: p. 419-431.

[18]. Lin, X., et al., Protective effect of madecassoside against cognitive impairment induced by d-galactose in mice. Pharmacology, Biochemistry and Behavior, 2014. 124: p. 434-442.

[19]. Bagheri, M., et al., Genistein ameliorates learning and memory deficits in amyloid $\beta(1-40)$ rat model of Alzheimer's disease. Neurobiology of Learning and Memory, 2011. 95(3): p. 270-276.

[20]. Ashe, K.H. and K.R. Zahs, Review: Probing the Biology of Alzheimer's Disease in Mice. Neuron, 2010. 66: p. 631-645. 